\title{
A landslide inventory system as a base for automated process and risk analyses
}

\author{
Thomas M. Kreuzer ${ }^{1}$ (D) $\cdot$ Martina Wilde $^{2} \cdot$ Birgit Terhorst $^{2} \cdot$ Bodo Damm $^{1}$
}

Received: 14 November 2016 / Accepted: 29 May 2017 / Published online: 11 July 2017

(C) The Author(s) 2017. This article is an open access publication

\begin{abstract}
Landslide research requires consistent and widespread data. Many countries within the European Union have national landslide inventories to fulfill these demands for their respective research. However, those inventories were usually not intended to provide the technical basis for automated process and risk analyses during their design phase. The ongoing development of Germany's national landslide database offers the opportunity to do this differently. This paper introduces a landslide inventory system called WISL suitable for data handling as well as for novel automated process and risk analyses on a national scale. WISL is designated to form the technical infrastructure for a German national database. Its core consists of an open source relational database management system (PostgreSQL), standardized input and registration methods as well as integrated analyses modules, which avoid large data movement and allow for rapid risk analyses. We present proof-of-concept
\end{abstract}

Communicated by: H. A. Babaie

Thomas M. Kreuzer

thomas.kreuzer@uni-vechta.de

Martina Wilde

martina.wilde@uni-wuerzburg.de

Birgit Terhorst

birgit.terhorst@uni-wuerzburg.de

Bodo Damm

bodo.damm@uni-vechta.de

1 Department of Applied Physical Geography, University of Vechta, Vechta, Germany

2 Institute for Geography and Geology, University of Würzburg, Würzburg, Germany results of endangered infrastructure related to automated risk mappings based on topography and proximity of active landslides. The use of open source software and the application of a standardized input and data acquisition system for experts, coupled with custom analysis modules, constitutes a step toward automated risk maps by a mere 'button-press'. Future developments for the inventory lie in the field of refining and inventing analysis modules and collecting data, for which WISL provides a firm technical base.

Keywords Database · Risk analyses · Landslides · Open source $\cdot$ Data scalability

\section{Introduction}

An accurate landslide inventory is a fundamental tool for all kinds of applied studies on landslides (Van Den Eeckhaut and Hervás 2012a). Registered information on landslide events plays a key role in landslide research. In general, risk assessment, disaster management as well as mitigation strongly demand for relevant datasets. Any statistical research depends on the availability of data. A more and more important requirement is the access to and distribution of data among different institutions and experts (Van Den Eeckhaut et al. 2012). Multifaceted tasks and analyses demand a highly flexible management for data archiving and distribution. An efficient tool to cover these multifaceted requirements of data analysis is a centralized database system connected to defined network users (Härder et al. 1988; Foster et al. 2011).

Efficient databases have to deal with a large amount of landslide records and data consistency is a highly relevant pre-condition. For that case, relational database management systems (RDBMS) software can keep data consistent 
and free of redundancy. The RDBMS has to maintain its performance with a permanently increasing amount of data while also serving the data to a Network (Yeung and Hall 2007). Yet, data access is only one side of a bi-directional data flow. As institutions can access registered landslide data, users should be able to insert data to the inventory analogously. Therefore, additionally to the RDBMS backend, an input frontend has to be available, whose data types and details of the registration process have to adapt to welldetermined standards for comparability and interoperability (Parsons 2011).

It is generally known that data acquisition on landslides is a time-consuming task. The RDBMS is thus required to operate in the long term. Low costs decrease the dependency on budget decisions and can therefore ensure a consistent uptime of the RDBMS. Furthermore, the system should be independent of the standing of a single company and allow for customizations without restrictions. Customization is necessary, because without it a permanent data growth causes an increase of costs and time for transfer and archiving operations. Therefore, analysis modules need to be directly integrated into the RDBMS to enable the transfer of analysis results, exclusively (Aye et al. 2016). Free opensource software can provide an infrastructure, which meets the aforementioned demands (Raymond 2001).

Primarily, this paper presents the technical implementation of a landslide RDBMS called 'Würzburg Information System on Landslides' (WISL) (Jäger et al. 2015). The system addresses three major pre-conditions for modern inventories i) consistent data storage and data distribution, ii) convenient, standardized data input and iii) module integration for highly flexible and rapid spatial analysis. It is therefore suitable for large scale application and allows for the operation of national inventories to avoid the short comings of regional databases, which are usually characterized by a restricted data access, different standards for data input, and strongly heterogeneous analysis capabilities and, of course, limited data sets (Damm and Klose 2014). Of the 22 EU member states with national landslide inventories some do not use a RDBMS at all, others operate commercial software solutions with their accompanying restrictions (Van Den Eeckhaut and Hervás 2012b). Thus, WISL presents a novel consolidation of various techniques and data sets for a national database of Germany (Damm and Klose 2015) based on open-source software.

WISL aims to decrease landslide inventory fragmentation, thus its target user base are experts of environmental organisations and universities. However, due to a heterogeneous background of data acquisition personal, non-experts shall be able to acquire the data as well. In this context, WISL supports the compilation of incomplete data sets. Additionally, established GIS procedures are integrated, so data input can be complemented by their calculated results.
In this case the data sheet fills the role of a ground-truth check list for the pre-calculated parts.

Furthermore, a WISL integrated proximity analysis related to local risks is illustrated in order to demonstrate a user application in the second part of this study. In this study, risk refers to objects of interest which are exposed to hazards, thus following the definition according to Ropeik (2002). The addressed example of a rapid output analysis serves for one of the main objective in risk research: it is a first step towards automatically generated risk maps.

\section{Technical implementation}

\section{Data storage and distribution}

The software structure of WISL is developed on the base of PostgreSQL. As an object relational database management system (ORDBMS), PostgreSQL is customized to process large amounts of complex data with numerous internal relations (Sandmeier et al. 2013). It provides high standards in terms of stability, capability, memory, and most important, the option of geodata processing. PostgreSQL stores single datasets in different tables, with operators establishing internal linkage. It is in synchronization with SQL (Structured Query Language) standards, providing all functions of SQL as a database language for generating, managing, and manipulating stored data as well as data queries. Although PostgreSQL supports handling geometric data, it is not capable to process and to store large volumes of spatial data. Therefore, PostGIS, an extension for PostgreSQL, was integrated into WISL. PostGIS does not only improve the storage of GIS data in the DBMS, but offers spatial operators, functions, data types, and indices for rapid spatial applications (Obe and Hsu 2011). Beside the creation of new geometric data, users may generate morphometric measurements and establish spatially related links between different objects (Obe and Hsu 2011; Mitchell et al. 2008). Furthermore, PostGIS enables editing, analyzing, and saving of spatial data (e.g. point, polylines or polygones) in a database without the usage of an external GIS. According to Obe and Hsu (2011), the extension of PostGIS transforms an object-relational DBMS into a spatial database.

The PostgreSQL software package contains a serverclient model, which distributes registered data as text. PostgreSQL/PostGIS is open source and free of charge. It can easily be accessed from external software tools. The nature of open-source guarantees independence of any single company over the development of the software package, yet, modifications and extensions are always possible (Raymond 2001), which is in turn also true for WISL. Virtually, all common open source GIS and analysis applications are able to handle and to visualize spatial information of a 
PostgreSQL/PostGIS database. Thus, WISL allows experts to access data by their own working tools from all over the world, e.g. the ' $\mathrm{R}$ software environment for statistical computing and graphics', a fact which is essential to strengthen the acceptance of WISL.

The integration of analytical tools into WISL demands the support of calculation intensive tasks by the hardware as well as the availability of appropriate storage space. Due to the 'in-database' approach, which means data and analysis modules are integrated and only queries and results are transferred, network speed is of minor importance and enables sophisticated analysis from mobile, or even slower connections.

\section{Data input}

One of the main requirements for all kinds of open database systems are standardized input data sets in order to assure reproducibility and statistical consistency. Additionally, a system with many participants has to ensure provenance of data (Tilmes et al. 2010).
On behalf of WISL, a main emphasize is placed on a detailed data acquisition in order to enable scientific analyses. This means, data is not supposed to come from second hand material, but as a result of field investigations. However, in practice this is often a reciprocal process: a second hand source (usually non-experts) gives a rough description of a landslide which is then further assessed by field observations. Data normalization of field information is realized with the help of the data sheet developed in the portable document format (PDF) (cmp. Fig. 1). The PDF allows for manual and digital data input to meet the objectives of landslide field surveys. Digitally, the sheet can be used on all devices supporting PDF input fields, this means nearly all currently available desktop computer or handheld devices. Furthermore, the data sheet is a conclusion of an iterative process of several years of landslide mappings and expert knowledge in exchange with federal authorities (Sandmeier et al. 2013).

The data sheet design follows a dualistic approach: on the one hand there are well-established factors of landslide susceptibility, respectively slope stability, as, for instance, slope

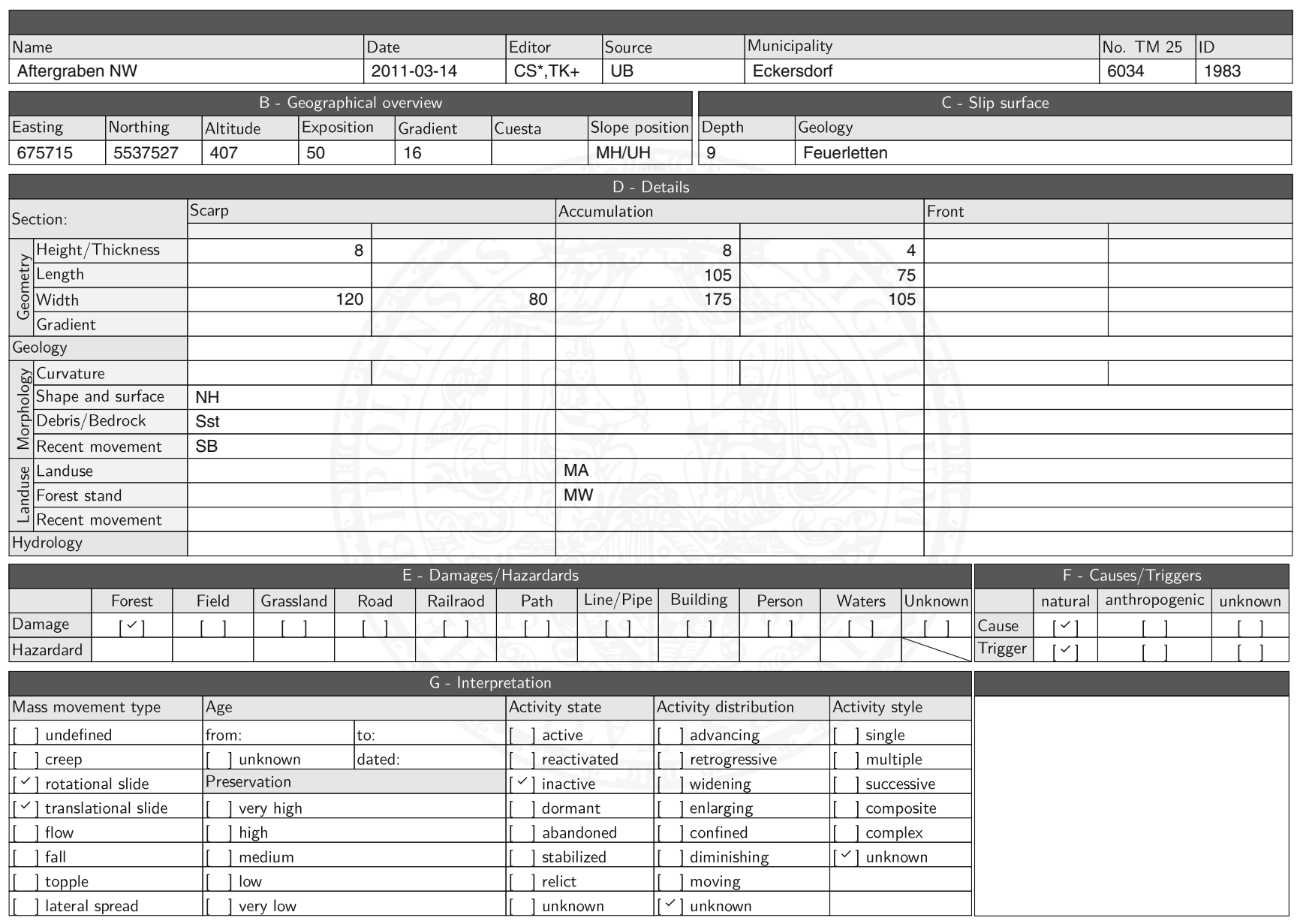

Fig. 1 Data sheet. PDF data sheet for standardized geomorphological data acquisition. The present data was acquired from a landslide near Ebermannstadt, Germany and information is filled in with abbreviations. The PDF includes forms for digital input and can be parsed directly into WISL, thus functioning as the user interface 
inclination, land cover, etc. Aleotti and Chowdhury (1999) and Dachroth (2002). On the other hand as much data as possible is collected for later statistical assessments. The latter approach is linked to the 'big data' concept (Lynch 2008; Provost and Fawcett 2013), which enables the data driven a-posteriori identification of more relevant landslide parameters. A notable characteristic of the developed data sheet is that it has records of raw data (Fig. 1, sections A - D) as well as interpreted data (Wieczorek 1984) (Fig. 1, sections E - H) for which expert knowledge is required (Jäger et al. 2015). In general, not all sections need to be filled in by the same person, thus a further practical approach is that raw data is acquired by non-experts, while the interpretative parts are added by an expert later on. Since the persons conducting the field survey are recorded as well (Fig. 1, section A), data provenance is satisfied and interpretations can be retraced.

The data sheet conforms one-to-one to the database structure and serves as WISL's input mask. Consequently, the data sheet provides a reference point (defined as the highest point on the scarp) of the landslide as spatial information (Fig. 1, section B). However, registered information for spatial descriptions of single landslide elements can be complemented with corresponding shapefiles. The respective elements are i) scarp, ii) accumulation and iii) front of the landslide and they are each mapped separately: scarp as a line shape, accumulation and front as polygons. The shapefiles can be provided in over 90 formats (GDAL Development Team 2017) and mobile GIS applications enable their creation during field work. The shape information can be imported into the database, but the original files are not stored in WISL. Due to the PostGIS extension WISL stores all mapped shapes as a further parameter of the related data set. This means that the shape corresponds to an attribute, like the name or the date. In case the software equipment supports spatial database access to PostgreSQL/PostGIS, the addition of the shapes can be processed directly at any time. Most open source software provide complete spatial access, however, closed source software, for instance, often prevents write access to PostgreSQL/PostGIS in order to favor their own competing products.

The data sheet contains some redundancy with the optional shapefiles, which enables non-GIS usage as well. More over, WISL offers the option to store photographs and drawings for archiving purposes.

The input of the PDF (data sheet) can either be processed by low level SQL commands or by a graphical user interface in form of a desktop GIS extension. A corresponding plug-in for common GIS software, namely ArcGIS and QGIS, was developed in order to reach convenience and better accessibility (Fig. 2). Additionally, the plug-in exposes WISL's ability to use PostGIS processing in order to calculate certain data values, e.g. curvature,slope steepness, land

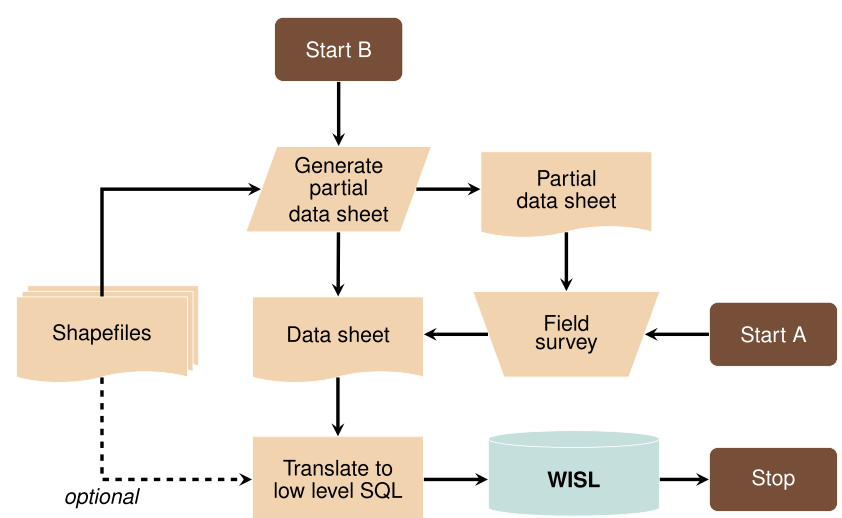

Fig. 2 Flow chart. Flow chart on how to enter data in the GIS plug-in with two separated entry points $\mathbf{A}$ and $\mathbf{B}$. A represents the work flow of an empty data sheet before field observations, shape information is optional. B represents the work flow with pre-calculated values in the data sheet, those pre-calculated values require shapefiles

cover, etc. These calculation procedures run with the help of auxiliary data within the database, e.g. digital elevation models and thematic maps. Optionally, if applied before the field survey, this extension can facilitate and serve field documentation: the data sheet fills the role of a ground-truth checklist for the pre-calculated values. Thus, the data sheet serves as input and output for the plug-in.

In case a landslide record is changed over time there are two procedures WISL can reflect the modification: i) attributes can simply be updated to the current values, ii) a secondary process can be linked to the main process. Both options imply that a new data sheet is submitted and WISL automatically creates a new modified data set. This means, that one landslide entry comprises two (or more) datasets. In each case the original one remains intact and is connected to the new dataset. During a query case i) only presents the latest dataset by default, case ii) provides all linked datasets.

\section{The automated risk identification module (ARIM)}

In general, it is possible to integrate analysis modules into WISL, which work entirely in the database to provide exclusively the requested analysis results. The following example serves as a proof-of-concept in order to demonstrate module integration.

Currently, digital methods for the creation of hazard and risk maps, e.g. SINMAP and ArcSDM (Neuhäuser and Terhorst 2007; Terhorst and Kreja 2009), demand manual adjustments for every area of interest. Disposition models have different requirements than process models thus the user needs to supply the required values. A visual example of a hazard map created by the infinite slope model (Montgomery and Dietrich 1994) with SINMAP is shown in Fig. 3. WISL accepts disposition and process models as integrated modules. In this context, ARIM follows the 


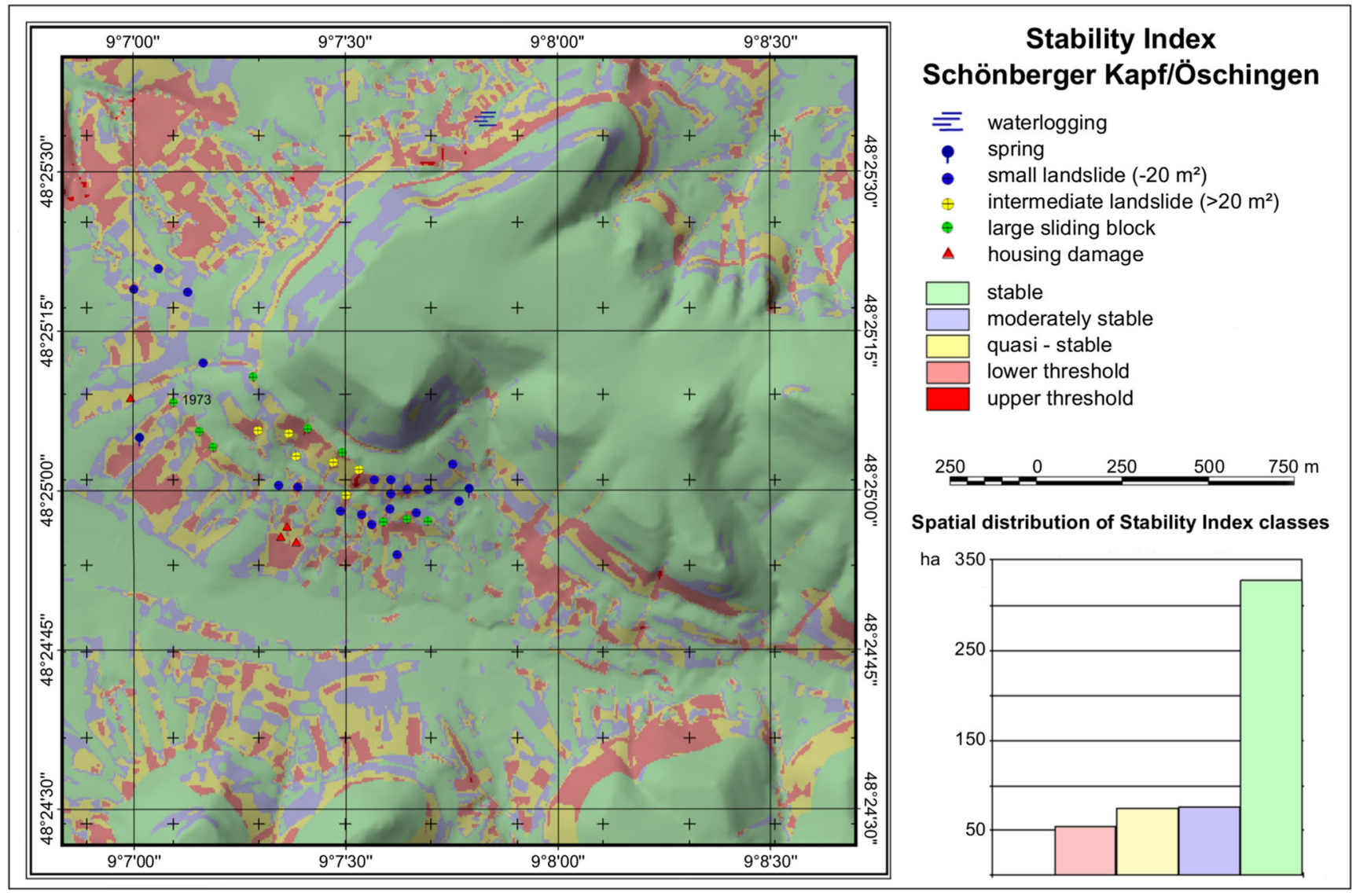

Fig. 3 Stability index map. Example of a stability index map of the Schönberger Kapf generated with SINMAP, a GIS extension modified after Terhorst and Kreja (2009)

objective of WISL to generate completely automated landslide susceptibility/risk maps. The module categorizes the hazard exposition in the proximity of landslides which were reported as active slope movements by field observation in the data sheet. Therefore, the module procedure calculates the area of possible flow direction of the active landslide and secondly, identifies exposed objects, e.g. buildings and infrastructure located in the flow path. For the successful execution of the ARIM the following data is required in WISL: i) location and extension of landslides, ii) activity state, iii) a digital elevation model (DEM), and iv) georeferenced infrastructure objects. Information on the location and extent as well as activity state are provided by the data sheet and its related shapefiles. The combination of DEM(s), e.g. SRTM data (Rabus et al. 2003), and open street map (OSM) data provide all further requirements. WISL stores all aforementioned information on the server unit, thus, applicants just need to send a query and only receive results, without any transfer of raw data. This procedure has the technical benefit of bandwidth usage reduction, allows for rapid access and can additionally avoid licensing issues, i.e. a licensed DEM.
For the module invocation it is necessary to send an SQL command to WISL with a user defined bounding box and minimum inclination. The results are object locations, which are endangered and situated within the defined bounding box. The necessary simple SQL command is defined as

\section{SELECT WISL_RiskAssessment(}

$$
\begin{aligned}
& \text { xll, -- lower left } x \text {-coordinate } \\
& \text { yll, -- lower left } y \text {-coordinate } \\
& \text { xur, -- upper right } x \text {-coordinate } \\
& \text { yur, -- upper right } y \text {-coordinate } \\
& \text { mingrad -- minimal inclination); }
\end{aligned}
$$

The module selects all active or potentially active landslide records in the database for a specified area of interest (xll, yll, xur, yur). It then rasterizes the respective accumulation area (slide mass). On the base of the newly generated raster data an outflow path is determined according to relief information from the DEM. Therefore, the module identifies the adjacent raster cells from an active 
landslide mass, which have an inclination equal or above the user defined level (mingrad). Subsequently, the outflow path is modeled in form of an arbitrarily shaped area, termed 'extended landslide flow area' (ELFA). All intersections of ELFA with georeferenced objects, like settlements and infrastructure, are marked. These modelling results are indicated as risk areas, where the identified objects are exposed to possible landslide damages from the (potentially) active mass.

Finally, the ARIM is a statistical process model, which means that the area of minimal inclination (ELFA) is selected empirically (see next chapter).

In PostgreSQL the module itself can be written in any programming language with a procedural language binding. The described implementation of ARIM is written in C and Python.

\section{Use case and testing: landslide flow extension}

In order to understand the integration and application of specialized modules (i.e. ARIM) from WISL, a use case related to flow direction is presented. The ARIM is an enhanced proximity hazard analysis based on preliminary research by Jäger et al. (2015). The module generates a first automated vulnerability assessment based on topography and is a step towards the production of automatically generated risk maps.

As a general rule, the following use case is also representative for arbitrary analytical modules similar to ARIM.

Description: Supplement landslide data within an area of interest and acquire objects susceptible to landslide damage

Actors: Expert, WISL, ARIM, GIS plug-in

Precondition: Landslide accumulation shape, OSM data and DEM must be stored in WISL for the requested area.

\section{Basic Course of Events:}

1. The expert/user identifies the area of interest.

2. The expert/user inserts landslide data for an area of interest by the GIS plug-in (optional).

3. The expert/user sends an SQL command to WISL to request objects susceptible to landslide damage within the area of interest.

4. WISL invokes the ARIM to identify potentially endangered objects and sends the result back to the expert/user.

5. Verification of the resulting hazard potential by the expert/user.

\section{Extensions:}

2a. Insert data directly via SQL.

3a. Adjust minimal inclination for path tracing.
In order to present first results of the ARIM, an area defined as the rectangle between the lower left coordinates of $11.4519^{\circ} \mathrm{E} 49.5273^{\circ} \mathrm{N}$ and the upper right coordinates of $11.5050^{\circ} \mathrm{E}$ and $49.5568^{\circ} \mathrm{N}$ was determined. The area is approximately $40 \mathrm{~km}$ east of Nuremberg in Germany. This area of interest comprises three (potentially) active landslide records in WISL, which the ARIM transformed by the help of a DEM into three ELFAs. A minimal inclination of $12^{\circ}$ was selected. This approach results in ELFAs up to twice as large as the actual accumulation area of landslides. The selected inclination is motivated by statistical analysis of slope cells next to the accumulation areas. The examined slope raster is generated by the available DEM. For this analysis 444 (semi-)active landslides of the Franconian Alb were investigated, these landslides had 8602 adjacent downward facing slope cells. The statistical analysis of those cells (Table 1) indicates that the majority of landslides (60\%) stopped in slope positions with inclinations lower than $11.7^{\circ}$. By consequence, a potential downslope advance is likely to occur at $12^{\circ}$.

As one relevant result, the ARIM identified an endangered railtrack in two of three ELFAs (Fig. 4). Expert knowledge, as documented in the manual data sheet, is in accordance with the automatically generated survey described above.

\section{Discussion}

WISL is a highly flexible and integrative database, which allows for easy registration procedures, data storage of general spatial information, statistical analyses, and custom made modules. Furthermore, it enables data transfers to external users on the base of results, without raw data movement, while currently many scientific data systems still exchange large amounts of data (Mattmann et al. 2016).

The structure of the landslide inventory is based on the open source software PostgreSQL/PostGIS. It corresponds to a low-budget-solution which provides independence of corporate support. The disadvantage is that there is no party with legal obligations for maintenance and warranty of operation. However, PostgreSQL/PostGIS is a tried-andtrue RDBMS deployed in many projects, which rely on the functioning of the software (PostgreSQL Featured Users 2016). Maintenance is thus a community effort potentially distributed among all users of the software. Projects in other

Table 1 Statistical results of downslope cells adjacent to landslide masses, $\mathrm{n}=8602$, numbers in degrees

\begin{tabular}{lllllll}
\hline Mean & Std. & Min. & Q1 & Median & Q3 & Max. \\
\hline 12.3 & 5.9 & 0.1 & 8.0 & 11.6 & 16.2 & 44.3 \\
\hline
\end{tabular}




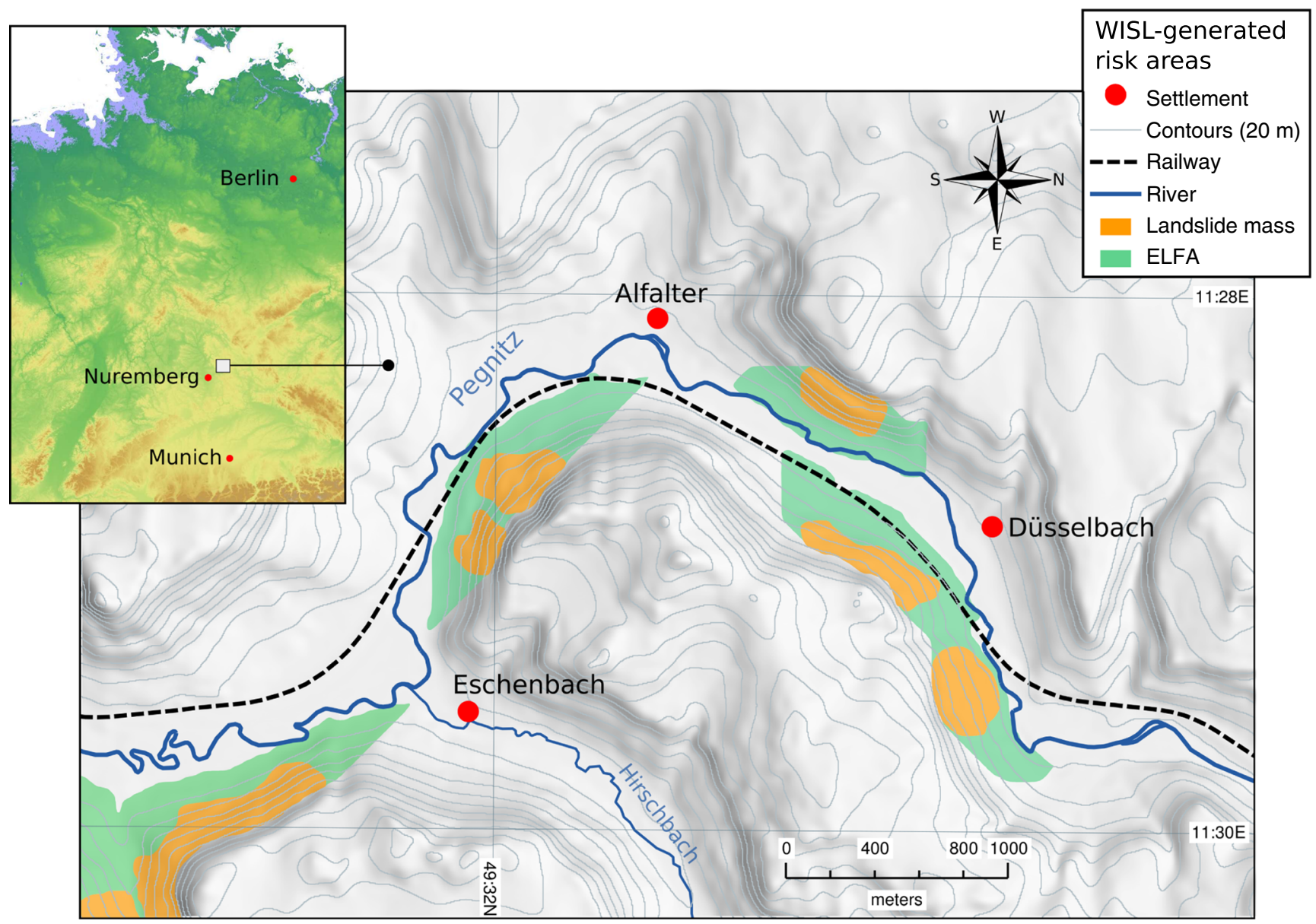

Fig. 4 Automated risk identification module. Railway between Nuremberg and Neuhaus (Pegnitz) in Northern Bavaria and the potential landslide runout (ELFA), with a minimum of $12^{\circ}$ inclination for

scientific fields have already good experience with open source databases (Craig et al. 2004). The competence of the system lies, in general, on its high flexibility, openness and integration capability for further modules.

The data sheet for landslide registration is mainly based on descriptions of field results and can be seen as an attempt to standardize landslide inventories. In this context, the data sheet defines the common denominator and additional shape information is optional which can lead to a heterogeneous data environment. In part, this problem is addressed through redundancy, i.e. shape and measurements of the landslide elements are documented in the data sheet and can be simultaneously derived from the optional shape. This also ensures that use of the data sheet is not restricted to GIS, but allows for instance physical archiving and manual analyses.

Even though, the focus is placed on German landslide sites, the registration sheet is designed to be transferable to further regions. Simultaneously, it serves as the digital user interface for the system, too. Besides information on measurable data, the possibility to record interpretative data is provided, which requires expert knowledge. the flow path, pictured on a shaded relief. The railway intersects the ELFA east of Düsselbach and west of Eschenbach and is thus marked as object at risk

Therefore, the system consists of a very detailed dataset. On the one hand, this approach allows for far-reaching as well as simple scientific analyses. On the other hand, the complexity might result in a disadvantage regarding objectives of end-users and can hamper widespread application. That is, because technical personal (e.g from forestry, road construction, etc.), which is commonly involved with landslide registrations, might not be able to handle interpretative data (Bechtold and Patterson 2005). However, data interpretation is not obligatory for the data input in WISL. Further development should target diverse approaches, from simple to complex.

The integration and application of custom analysis modules (i.e. ARIM) extended the capabilities of WISL to a great extent. The elucidated case study shows that rapid spatial analyses of registered data is possible. The ARIM as a topographic analysis module can be seen as one step towards automatically generated risk analyses. Although the latter is still a future objective, which needs further research, the ARIM already excludes false positives for objects endangered by landslides. In terms of validity it is a 
lower border of confidence (Chung and Fabbri 2003). This is meant to decrease further efforts, if a more detailed risk analysis is requested. In this context, ARIM is applied in order to expose challenges of the selected approach related to automated analyses (Wieczorek 1984; van Westen et al. 2008). The decision to implement the ARIM as a statistical model coupled with empirical expert knowledge was made due to the unavailability of physical parameters for a wide area of interest. Yet, WISL is designed for data scalability: as additional information is obtained, e.g. reholitic parameters, lithographic maps etc., respective models can complement ARIM. Thus, WISL delivers a common base for statistically and physically based disposition and procedural modelling.

There is a mandatory necessity to enable complex and rapid hazard analyses for landslides. Therefore, models like the ARIM can be part of a tool set aimed to generate automated process/risk maps. Further improvements can be provided by reliable statistical disposition models for landslides, e.g. weights-of-evidence (Bonham-Carter et al. 1989). A long term goal should aim to enable a mere 'button press' in order to create hazard maps. This goal is reached in part by complementary data sets, like DEMs, geological maps, etc. WISL thereby ensures that raw data is not transferred and thus reduces data movement and can avoid distribution restrictions of the copyright holder at the same time.

All in all WISL represents a system mainly valid for landslide experts and it is open for further extensions related to rapid susceptibility, hazard and risk analyses. It is thus an ideal technical basis for large scale projects like national landslide inventories. It's strength lies in the provenance (Tilmes et al. 2010) and consolidation of usage, mapping and analytical components. It's open source and distribution model allow for low-cost and long term operation. The data handling capabilities are only restricted by hardware and comparable to any commercial solution. If the datasets of the currently developing national landslide inventory for Germany and the technical foundation of WISL are combined, a new and viable tool for landslide research is available (Damm and Klose 2015).

Acknowledgements We are very grateful for the funding of the German Research Foundation DFG (projects: Te295/9-1 and 9-2, DA 452/5-1) and the Lower Saxonian Mininstry for Science MWK (projects: MWK 76ZN1504, 2016-2019 - "Niedersächsisches Vorab"). The input of the former team at the university of Würzburg supported the studies very much and we thank Christine Sandmeier, Daniel Jäger, and Stefan Bemm.

\section{Compliance with Ethical Standards}

Competing interests The authors declare that they have no competing interests.
Open Access This article is distributed under the terms of the Creative Commons Attribution 4.0 International License (http:// creativecommons.org/licenses/by/4.0/), which permits unrestricted use, distribution, and reproduction in any medium, provided you give appropriate credit to the original author(s) and the source, provide a link to the Creative Commons license, and indicate if changes were made.

\section{References}

Aleotti P, Chowdhury R (1999) Landslide hazard assessment: summary review and new perspectives. Bull Eng Geol Environ $58(1): 21-44$

Aye ZC, Jaboyedoff M, Derron MH, van Westen CJ, Hussin HY, Ciurean RL, Frigerio S, Pasuto A (2016) An interactive web-GIS tool for risk analysis: a case study in the Fella River basin, Italy. Nat Hazards Earth Syst Sci 16:85-101

Bechtold WA, Patterson PL (2005) The enhanced forest inventory and analysis program - national sampling design and estimation procedures. Gen. Tech. Rep. SRS-80, U.S. Department of Agriculture, Forest Service, Southern Research Station, Asheville $\mathrm{NC}$

Bonham-Carter GF, Agterberg FP, Wright DF (1989) Weights of evidence modelling: a new approach to mapping mineral potential. Stat Appl Earth Sci 89(9):171-183

Chung C-JF, Fabbri AG (2003) Validation of spatial prediction models for landslide hazard mapping. Nat Hazards 30:451-472

Craig R, Cortens JP, Beavis RC (2004) Open source system for analyzing, validating, and storing protein identification data. J Proteome Res 3(6):1234-1242

Damm B, Klose M (2014) Landslide database for the federal republic of Germany: a tool for analysis of mass movement processes. In: Sassa K, Canuti P, Yang Y (eds) Landslide science for a safer geoenvironment. Springer, Berlin, pp 787-792

Damm B, Klose M (2015) The landslide database for Germany: Closing the gap at national level. Geomorphology 249:82-93

Dachroth WR (2002) Handbuch der Baugeologie und Geotechnik, 3rd edn. Springer, Heidelberg

Foster C, Pennington CVL, Culshaw MG, Lawrie K (2011) The national landslide database of Great Britain: development, evolution and applications. Environ Earth Sci 66(3):941-953

GDAL Development Team (2017) GDAL - Geospatial data abstraction library, version 2.1.3. Open Source Geospatial Foundation. Open Source Geospatial Foundation. http://www.gdal.org

Härder T, Hübel C, Meyer-Wegener K, Mitschang B (1988) Processing and transaction concepts for cooperation of engineering workstations and a database server. Data Knowl Eng 3(2):87-107

Jäger D, Kreuzer T, Wilde M, Bemm S, Terhorst B (2015) A spatial database for landslides in northern Bavaria: a methodological approach. Geomorphology. doi:10.1016/j.geomorph.2015.10.008

Lynch C (2008) Big data: How do your data grow? Nature 455:28-29

Mattmann CA, Cinquini L, Zimdars P, Joyce M, Khudikyan S (2016) A topical evaluation and discussion of data movement technologies for data-intensive scientific applications. Earth Sci Inf $9(2): 247-262$

Mitchell T, Christl A, Emde A (2008) Web-mapping Mit Open sourceGIS-tools. O'Reilly Köln

Montgomery DR, Dietrich WE (1994) A physically based model for the topographic control on shallow landsliding. Water Resour Res 30(4):1153-1171

Neuhäuser B, Terhorst B (2007) Landslide susceptibility assessment using "weights-of-evidence" applied to a study area at the jurassic escarpment (sw-Germany). Geomorphology 86(1-2):12-24 
Obe RO, Hsu LS (2011) PostGIS in action. Manning Publications, Greenwich (Connecticut)

Parsons MA (2011) Making data useful for modelers to understand complex earth systems. Earth Sci Inf 4(4):197-223

PostgreSQL Featured Users (2016) Retrieved October 11th. https:// www.postgresql.org/about/users/

Provost F, Fawcett T (2013) Data science and its relationship to big data and data-driven decision making. Big Data 1(1):5159

Rabus B, Eineder M, Roth A, Bamler R (2003) The shuttle radar topography mission - a new class of digital elevation models acquired by spaceborne radar. ISPRS J Photogramm Remote Sens 57(4):241262

Raymond ES (2001) The Cathedral \& the Bazaar. Musings on Linux and open source by an accidental revolutionary. O'Reilly, Cambridge

Ropeik D (2002) Risk: a practical guide for deciding what's really safe and what's really dangerous in the world around you. Houghton Mifflin Harcourt, Wilmington

Sandmeier C, Büdel C, Schwindt D (2013) Multi-methodological investigation of a mass movement in the cuesta landscape of the Northeastern Franconian Alb, Germany. In: EGU general assembly conference abstracts, vol 15. Vienna, p 3298. EGU2013-3298
Terhorst B, Kreja R (2009) Slope stability modelling with sinmap in a settlement area of the swabian alb. Landslides 6(4):309-319

Tilmes C, Yesha Y, Halem M (2010) Tracking provenance of earth science data. Earth Sci Inf 3(1):59-65

van Westen CJ, Castellanos E, Kuriakose SL (2008) Spatial data for landslide susceptibility, hazard, and vulnerability assessment: an overview. Eng Geol 102(3-4):112-131

Van Den Eeckhaut M, Hervás J (2012a) Landslide inventories in Europe and policy recommendations for their interoperability and harmonisation. Technical report, European Comission, Ispra, Italy

Van Den Eeckhaut M, Hervás J (2012b) State of the art of national landslide databases in Europe and their potential for assessing landslide susceptibility, hazard and risk. Geomorphology 139140:545-558

Van Den Eeckhaut M, Hervás J, Jaedicke C, Malet J-P, Montanarella L, Nadim F (2012) Statistical modelling of europe-wide landslide susceptibility using limited landslide inventory data. Landslides 9:357-369

Wieczorek GF (1984) Preparing a detailed landslide-inventory map for hazard evaluation and reduction. Environ Eng Geosci 21(3):337342

Yeung AKW, Hall GB (2007) Spatial database systems. Springer, Dordrecht 